\title{
SIMBIOSYS-STC ready for launch: a technical recap
}

Emanuele Simioni

V. Da Deppo, C. Re, M. T. Capria, G. Naletto, G. Forlani, L. Tommasi, M. Dami, D. Borrelli, I. Ficai Veltroni, M. Massironi, A. Slemer, R. Mugnuolo, F. Longo,

Gabriele Cremonese 


\section{Bepicolombo Context}

\section{Launch:}

20 October 2018, 4:00 a.m [Rome]

\section{Arrival:}

5 December 2025

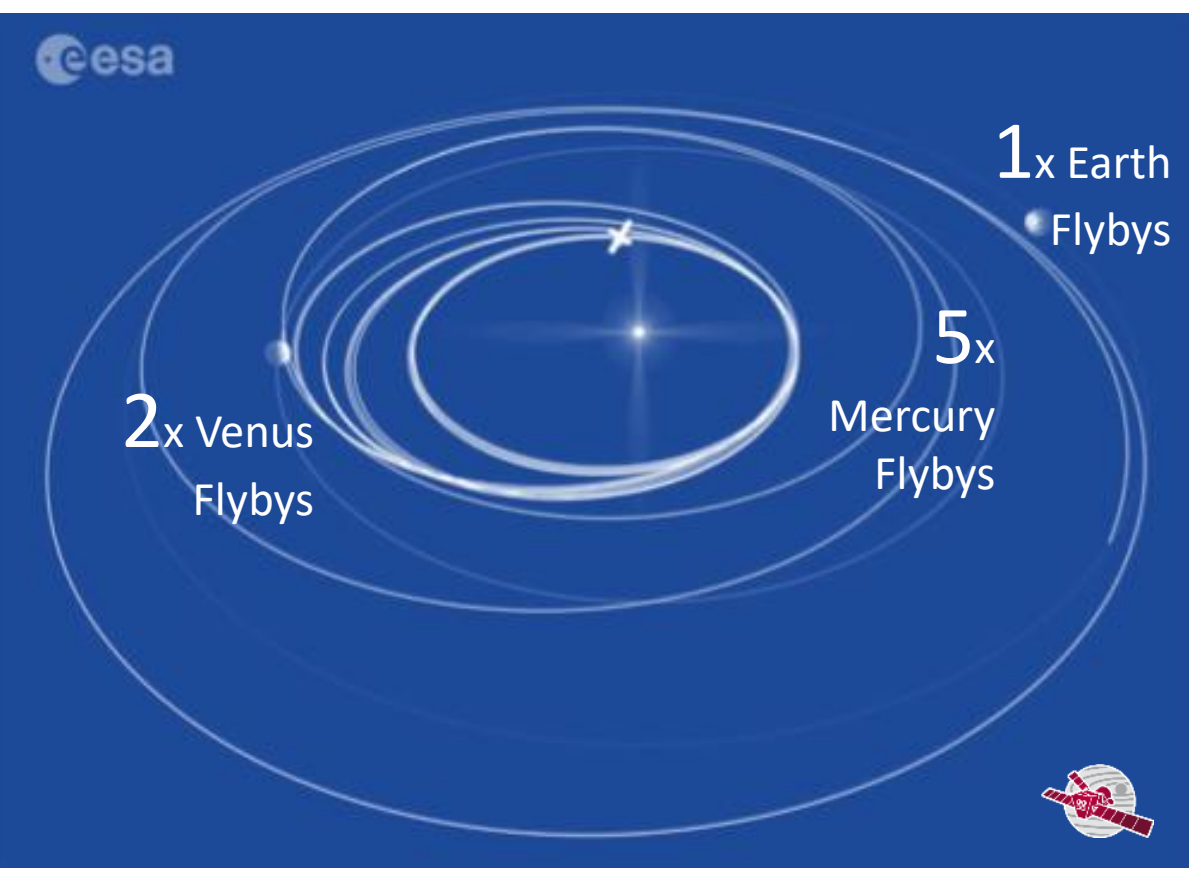

Timeline:

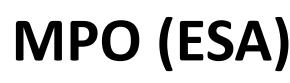

-NECP (Near earth commissioning phase)

-Cruise phase

-Commissioning Phase

-Mercury scientific phase

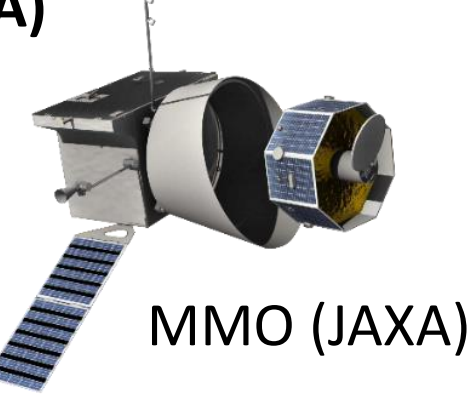

NECP

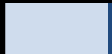

SCIENTIFIC 


\section{Mass:}

Solar Panel:

$1180 \mathrm{Kg}$

1300 Watt

Data Storage:

$47 \mathrm{~GB}$

Fuel:

Payload:

-11 Instruments -Scientific Regións:

- General relativity

- Magnetosphere

- Exosphere

- Internal Structure

- Surface

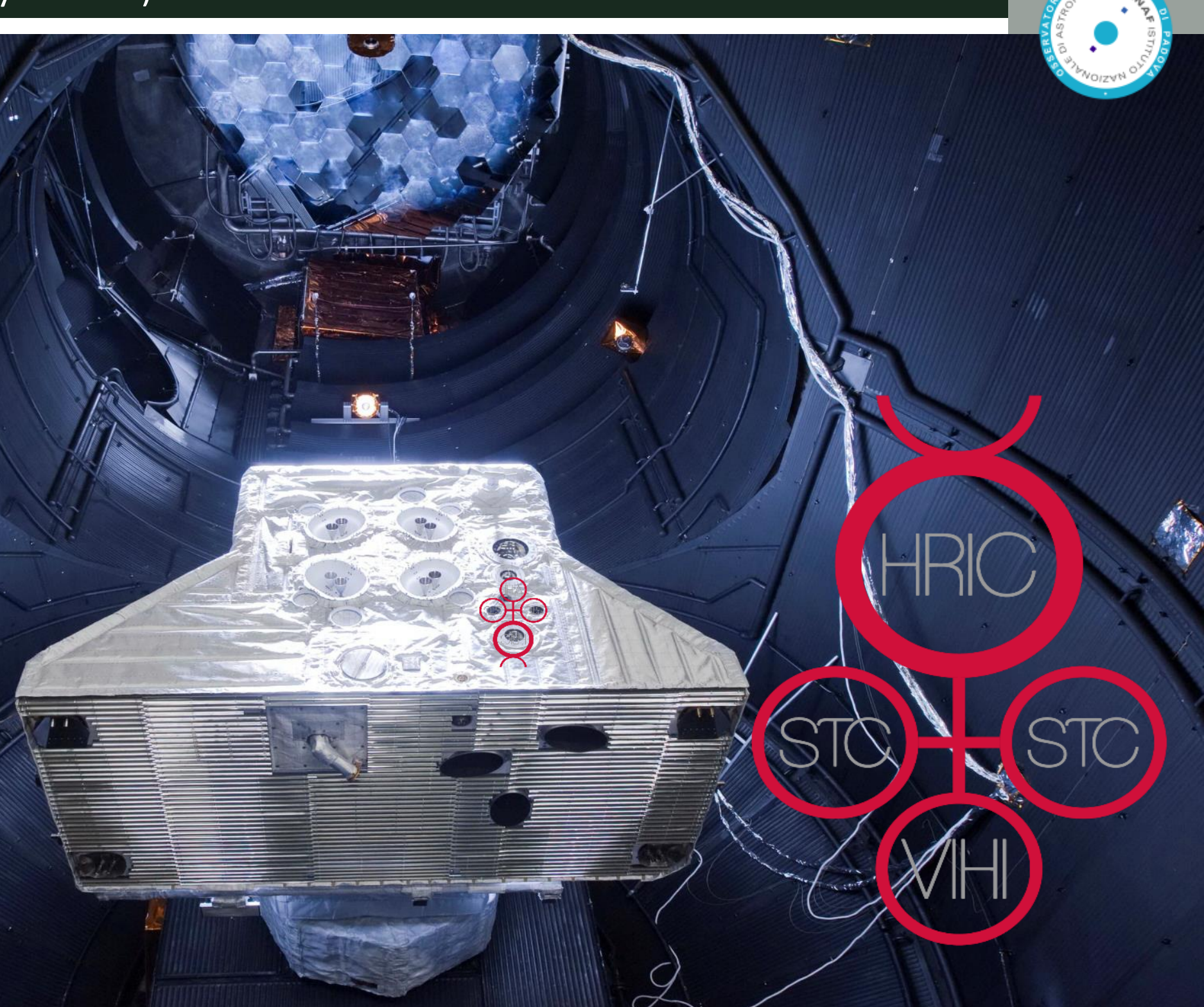

@ Large Space Simulator (ESA CREDITS)

(.) ब बip $\mathbb{E}$ 


\section{SIMBIO-SYS Spectrometers and Imagers for MPO \\ BepiColombo Integrated Observatory SYStem}

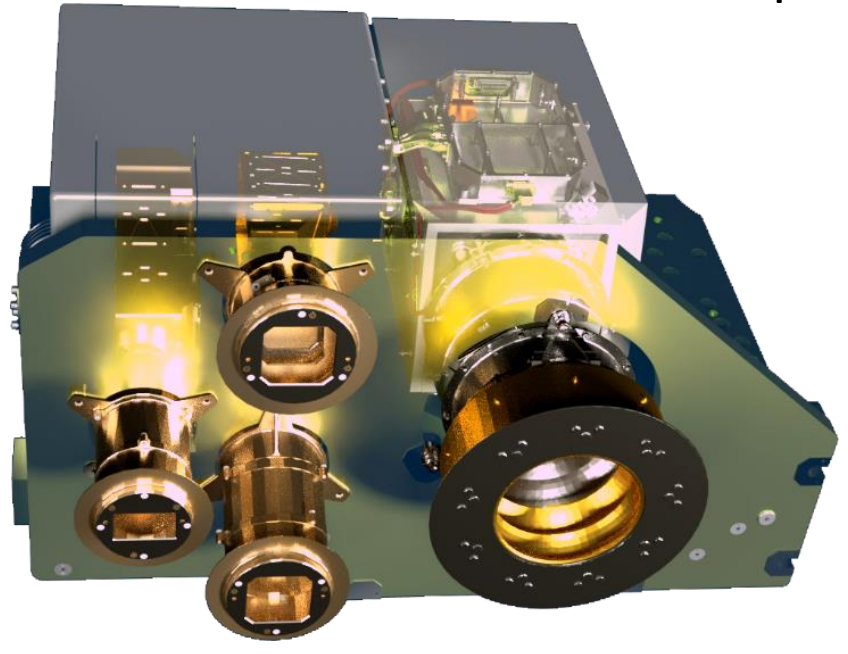

Industry Prime: $\quad$ "I $\angle E \square N A R \square \square$

Principal Investigator

Gabriele Cremonese (INAF, OAPd)

Co-P.I.

Fabrizio Capaccioni (INAF, IAPS)

Pasquale Palumbo (Università Parthenopea, Naples)

Maria Teresa Capria (INAF, IAPS)

Yves Langevin (IAS, Orsay)

Alain Doressoundiram (LESIA, Observatoire de Paris)

Technical Manager

Stefano Debei (CISAS, Padua)

"Simbiotic" electrical architecture: dedicated PE common ME (13 kg)

- HRIC(High-Resolution Imaging Channel)

- VIHI (Visible Infrared Hyperspectral Imager)

- STC (STereo Channel)

All suite: $13 \mathrm{~kg}$. 

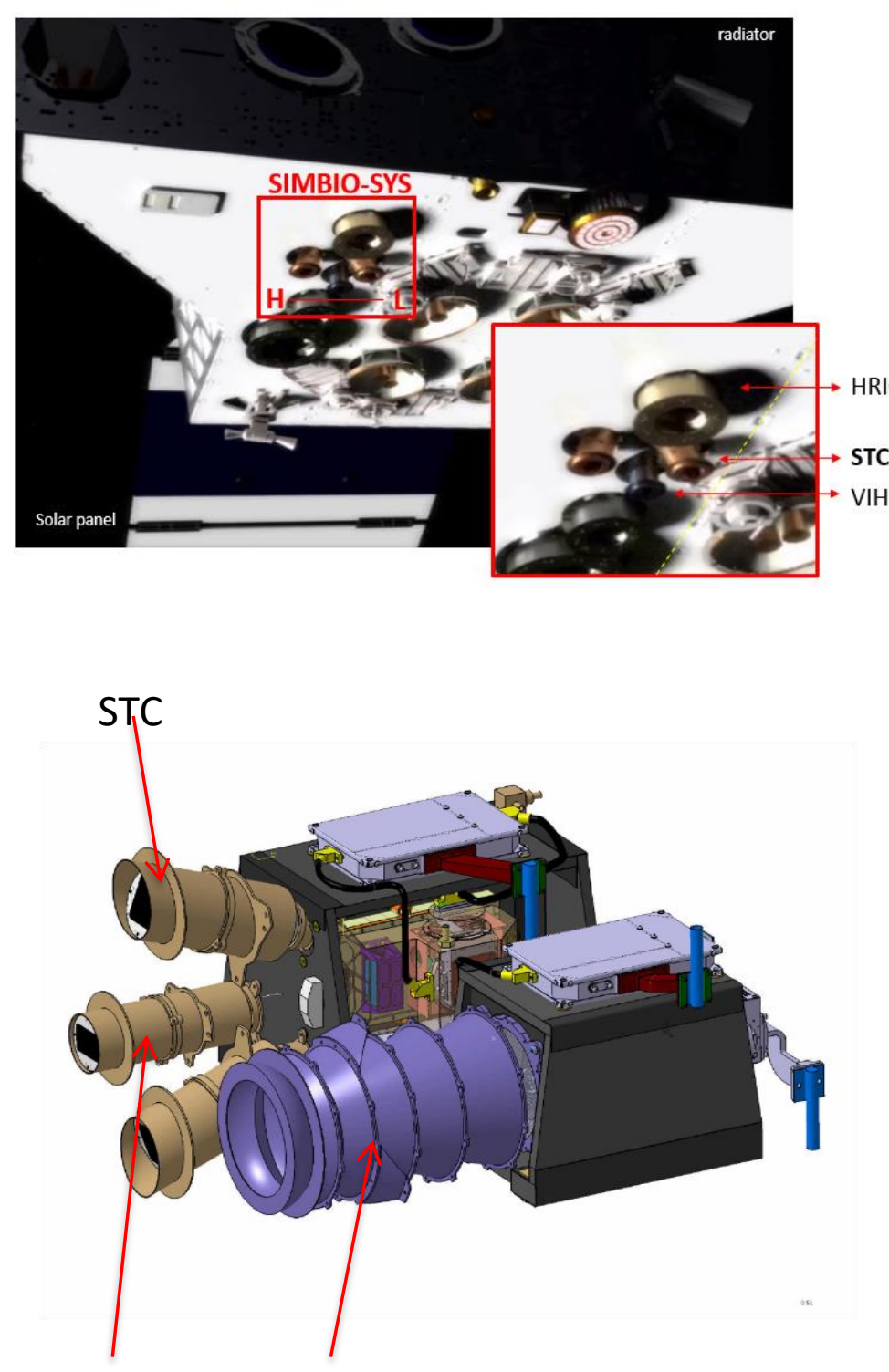

VIHI

HRIC
STC

Global stereo mapping and DTM reconstruction.

Spatial scale 60 m/pixel @ periherm (120 @poles).

Vertical Accuracy: 80 m @ periherm

Filters: pan, 420, 550, 750, 920 nm.

\section{HRIC}

Local mapping at high resolution with colour capabilities.

Spatial scale $6 \mathrm{~m} /$ pixel @ periherm Filters: pan, 550, 750, $880 \mathrm{~nm}$.

\section{VIHI}

Global mineralogical mapping;

Spatial scale 120 m/pixel @ periherm Spectral coverage 0.4-2.0 $\mu \mathrm{m}$ with $6.25 \mathrm{~nm}$ resolution
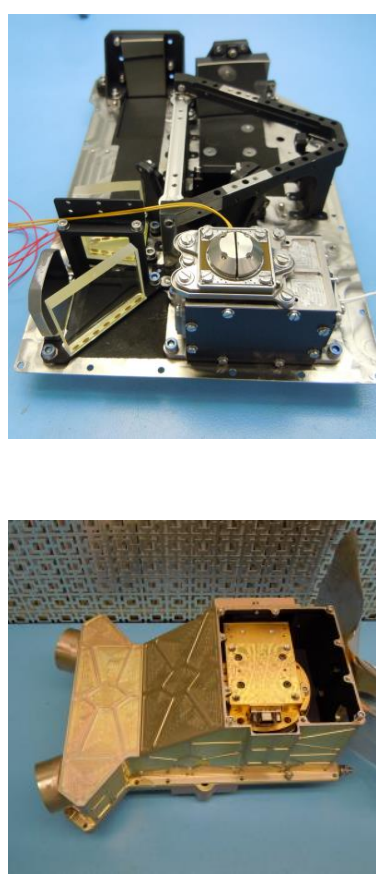

\section{量}


Timeline: 6M (Global Mapping) + 6M (Color Mode) + $1 Y$ (extention)
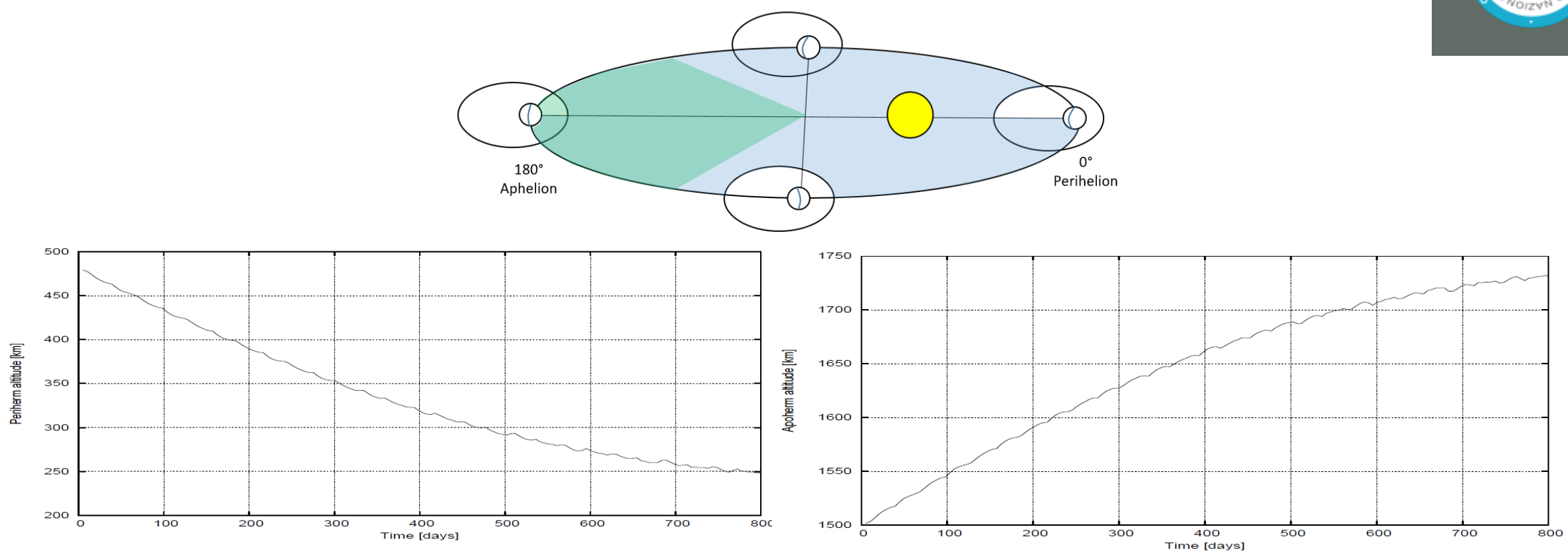

Orbit:

- MPO orbit is polar and 3 axis stabilized

- $480 \times 1500$ to $250 \times 1730 \mathrm{~km}$

- The periherm argument will change from $+16^{\circ}$ to $-40^{\circ}$.

STC Nominal Acq (+-42 ${ }^{\circ}$ TA)

CrossTrack overlapping 50\% @periherm

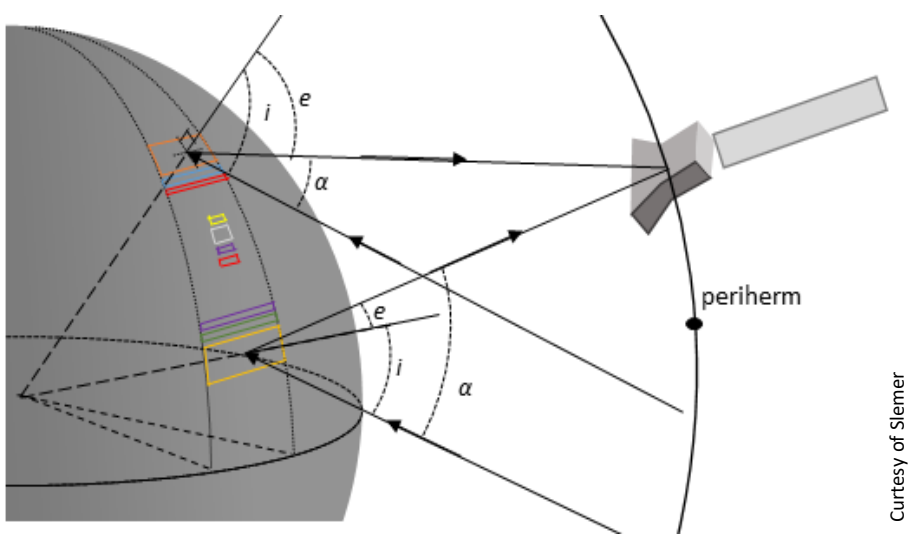

So changing radiance scenario makes the operations a delicate task. 


\section{Objectives}

- Global mapping in stereo mode

- Local mapping (Stereo and color)

Spice kernel predictions:

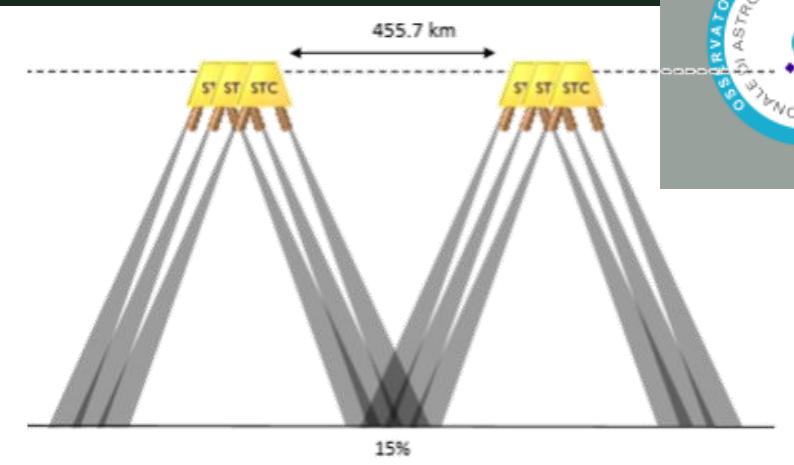

\section{Global Mapping}

\section{Periherm Poles}

\begin{tabular}{cc|cc}
\hline \hline S/C Height & {$[\mathrm{km}]$} & 480 & 966.7 \\
PoG & {$[\mathrm{m}]$} & 58 & 121.8 \\
Swath CT & {$[\mathrm{km}]$} & 48.3 & 100.96 \\
& Lat $\left[^{\circ}\right]$ & 1.19 & 7.9 \\
Swath AT & {$[\mathrm{km}]$} & 23.3 & 52.5 \\
& Long $\left[^{\circ}\right]$ & 0.55 & 1.2
\end{tabular}

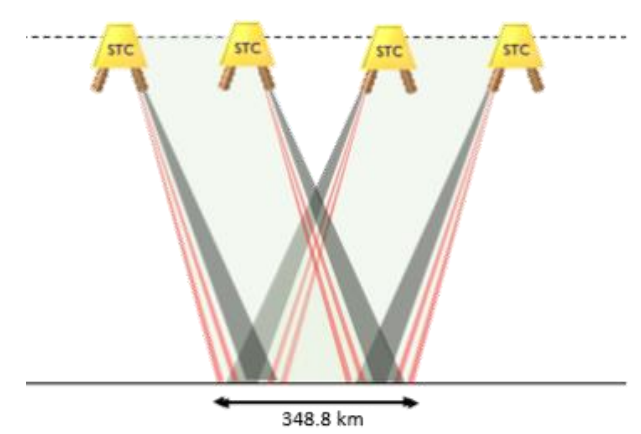

Color Mode

Flight Operation Procedures (FOPs).

These can be grouped into 4 distinct types depending on the application context:

- Flight Control Procedure (FCP)

- ENGineering procedure (ENG)

- CRitical Procedure (CRP)

- TeST procedure (TST)

\begin{tabular}{|lrl|}
\hline \multicolumn{2}{c|}{ FOPs parameters } \\
Name & Description & Limits \\
\hline Nacq & number of acquisitions & Nacq $<65535$ \\
Nw & number of windows & $0<\mathrm{Nw}<7$ \\
Wc & windows coordinates & filters depending \\
CT & cross track window dimension & CT $>128$, multiple of CBD \\
CBD & compression box dim. & $64 \times 64$ or $64 \times 128$ \\
IT & integration time & $(400 \mathrm{~ns}<\mathrm{IT}<10 \mathrm{~s})$ \\
IBR & inverse bit rate & $0 / 1$ lossless, $1<\mathrm{IBR}<64$ lossy \\
RT & repetition time & $\mathrm{RT}>150 \mathrm{~ms}$ \\
Priority & number of high priority acquisitions & $\mathrm{P}<255$ \\
\hline
\end{tabular}




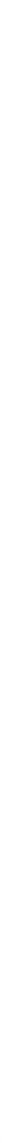




\section{Stereo Cameras}
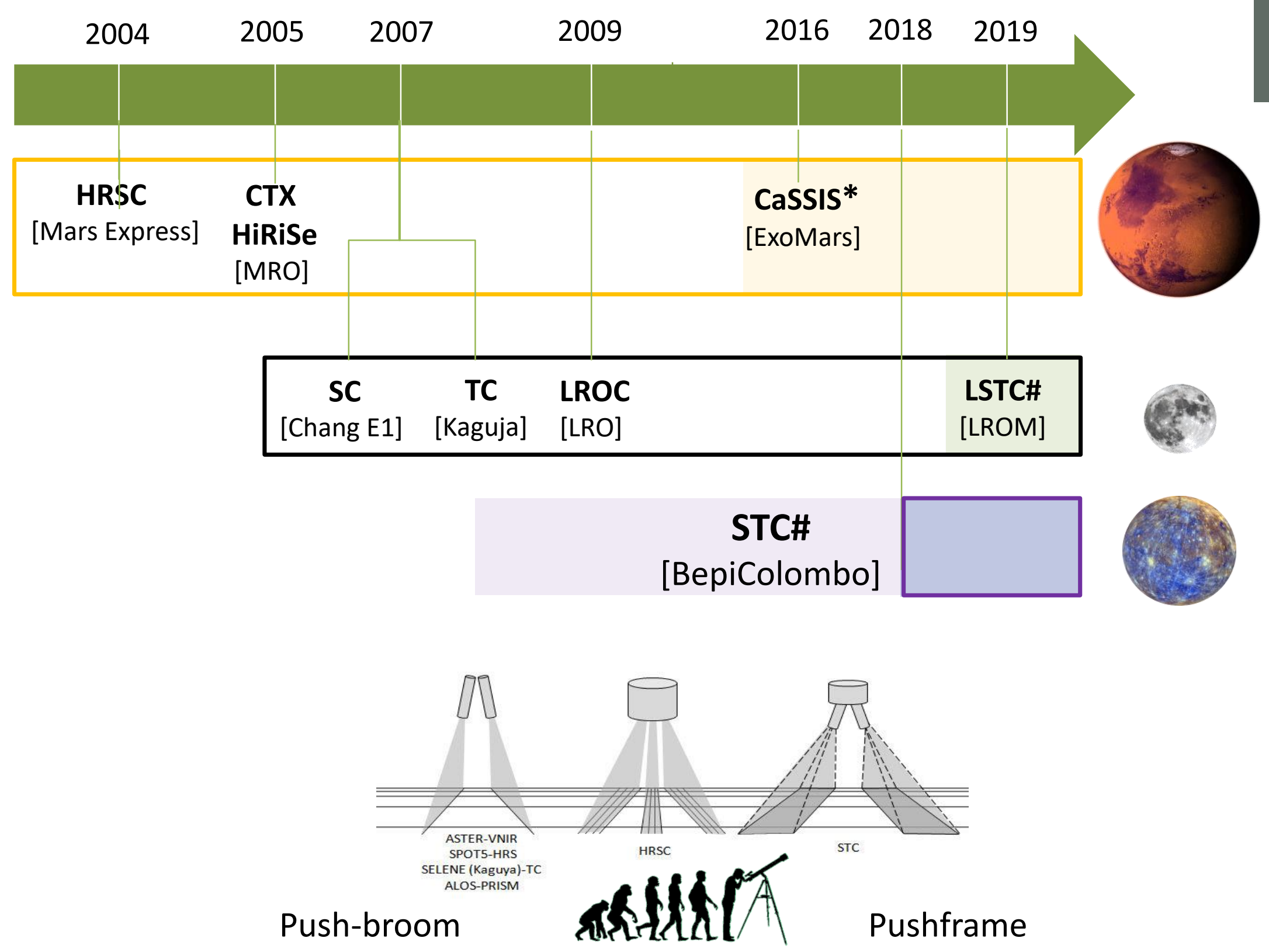

Push-broom

Pushframe 


\section{Focal Plane Assembly}

Cross track

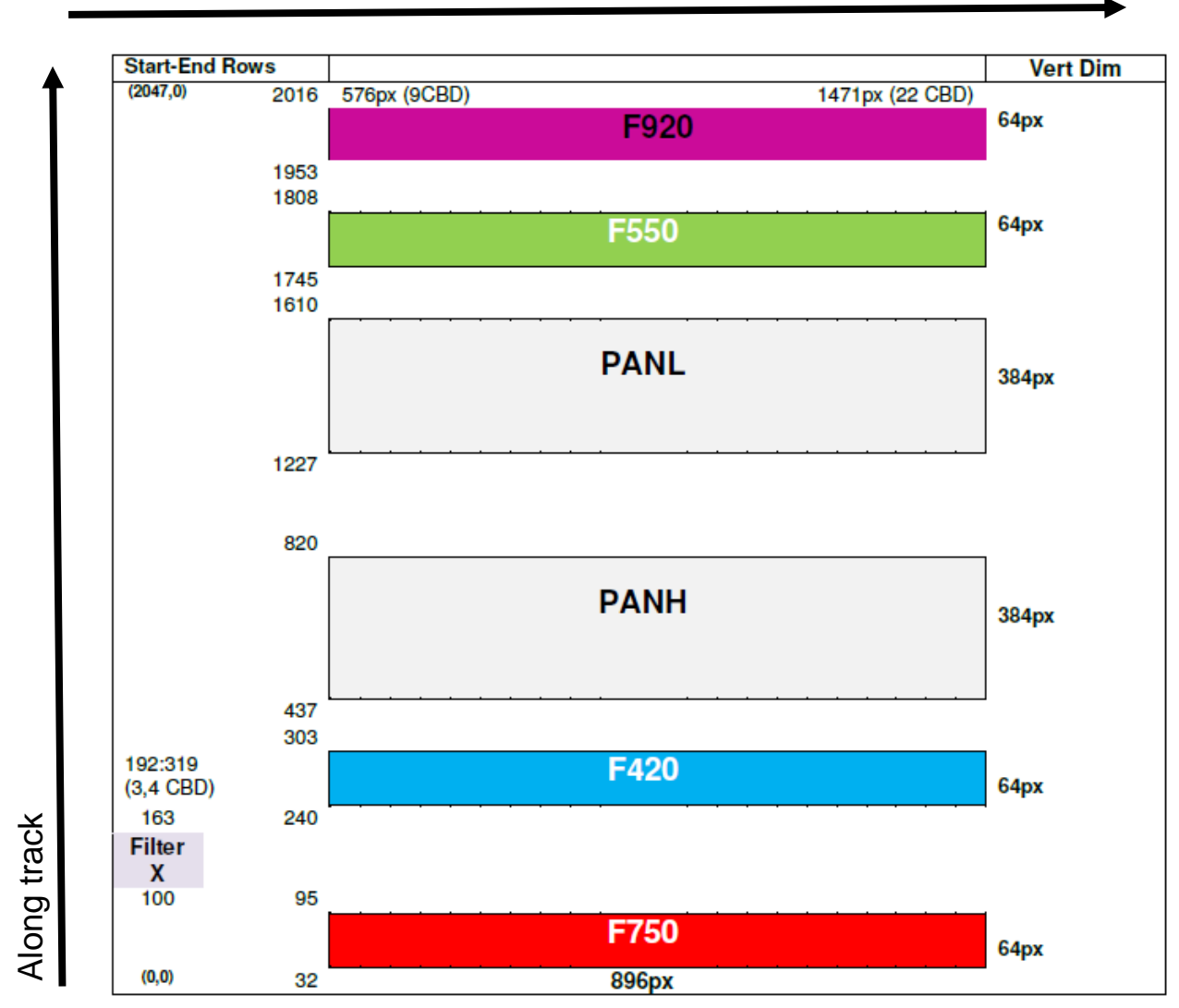

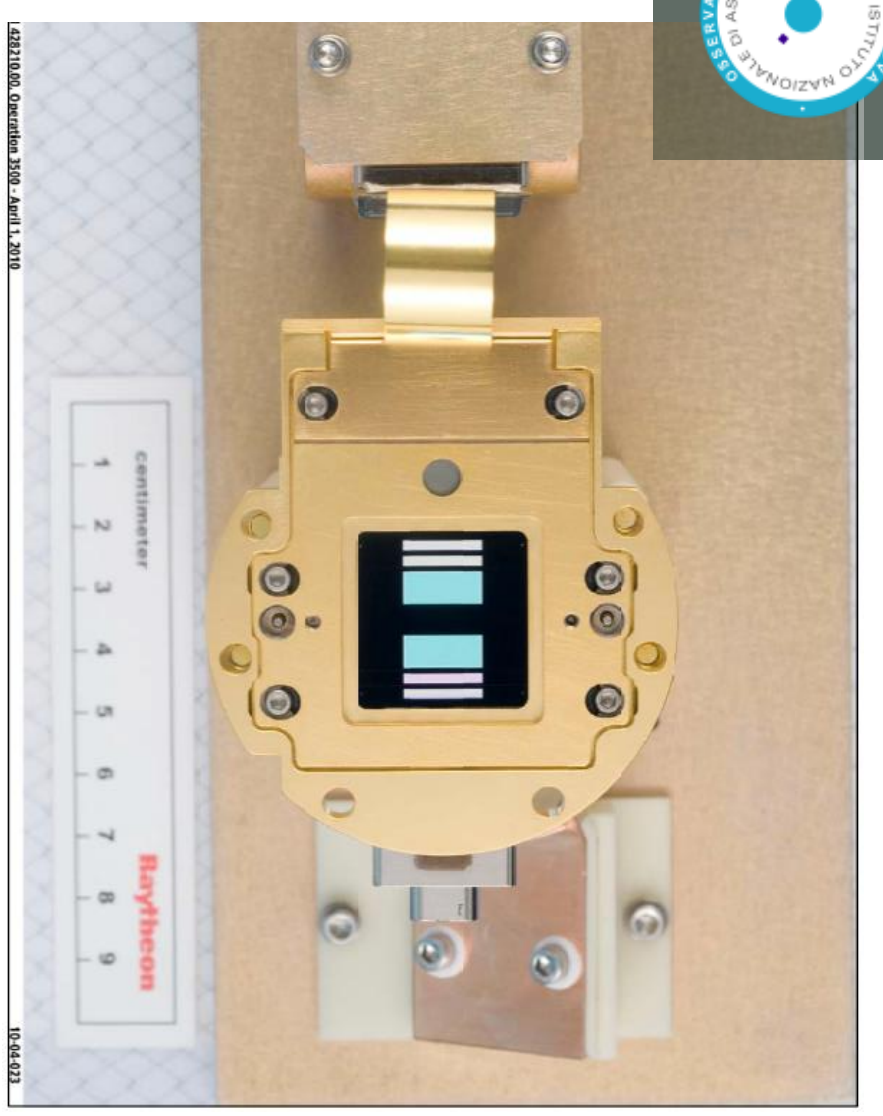

\begin{tabular}{|l|c|l|c|c|c|}
\hline Sensor dimensions & $2048 \times 2048 \mathrm{px}^{2}$ & Fill Factor & $100 \%$ & Read out Noise (RMS) & $60 \mathrm{e}-$ \\
\hline Unit Cell & $10 \times 10 \mu \mathrm{m}^{2}$ & Spectral Flatness & $8 \%(@ \mathrm{NIR})$ & $10 \mathrm{DN}(@ 268 \mathrm{~K})$ \\
\hline Min. Window & $128 \times 64 \mathrm{px}^{2}$ & Quantum efficiencies & $92 \%$ & Charge conversion & $14 \mu \mathrm{V} / \mathrm{e}-$ \\
\hline Max. N windows & 6 windows & FPA power budget & $120 \mathrm{~mW}$ & Integration Time range & 0.04 to $5000 \mathrm{~ms}$ \\
\hline A/D Conversion & $14 \mathrm{bit}$ & Analog output velocity & $<5 \mathrm{Mpps}$ & Operating temperature & $263-287 \mathrm{~K}$ \\
\hline
\end{tabular}




\section{Calibration Setup (Optical Ground Support Equipment (OGSE)}
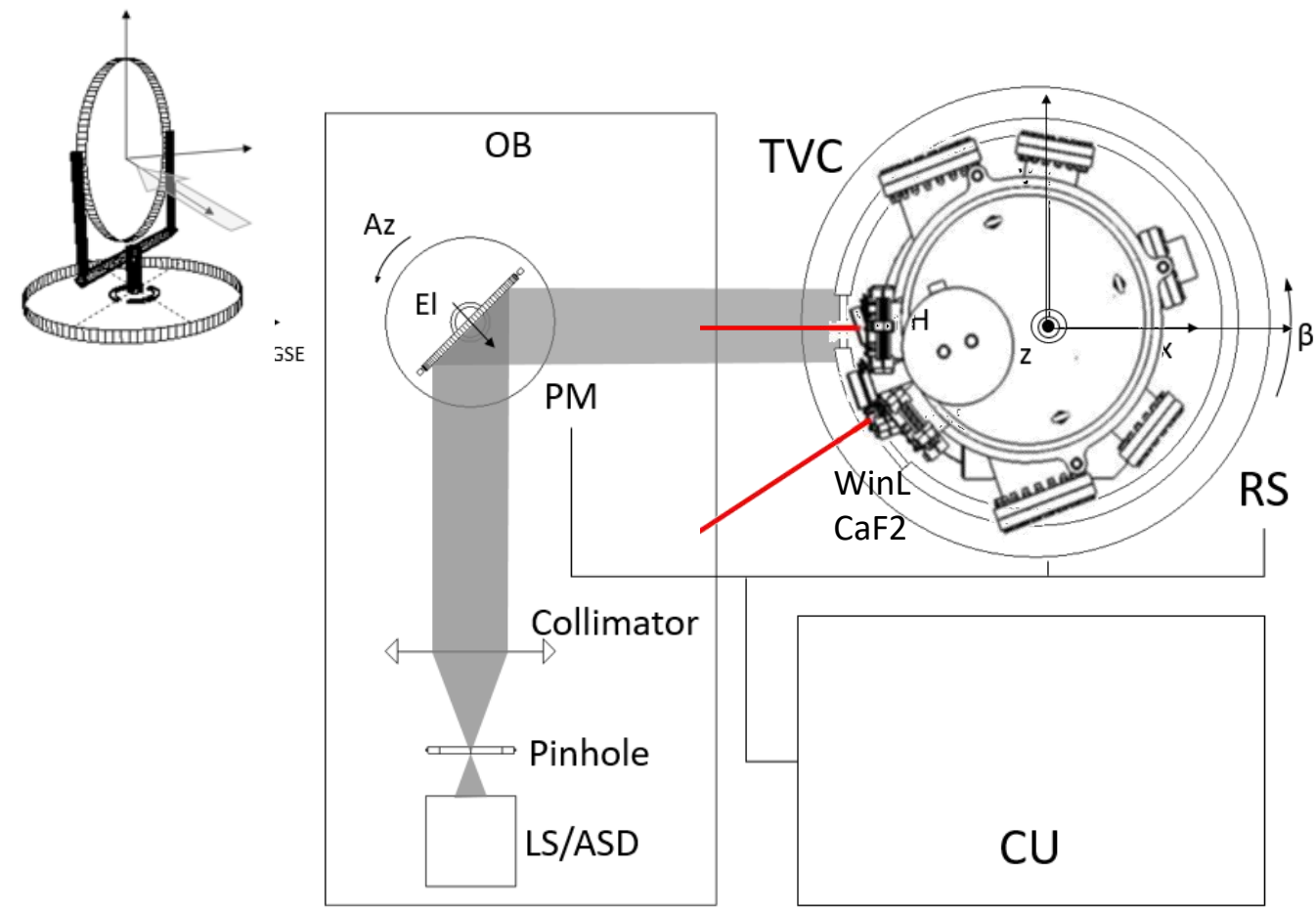

The optical system consists in:

- different light interchangeable source

- a collimator lens chromatically corr. $f=750 \mathrm{~mm}$

- a suitable target (either a pinhole or a diffuser)

- a rotating plane folding mirror

- a TVC (3w CaF2) mounted on a rotational stage.

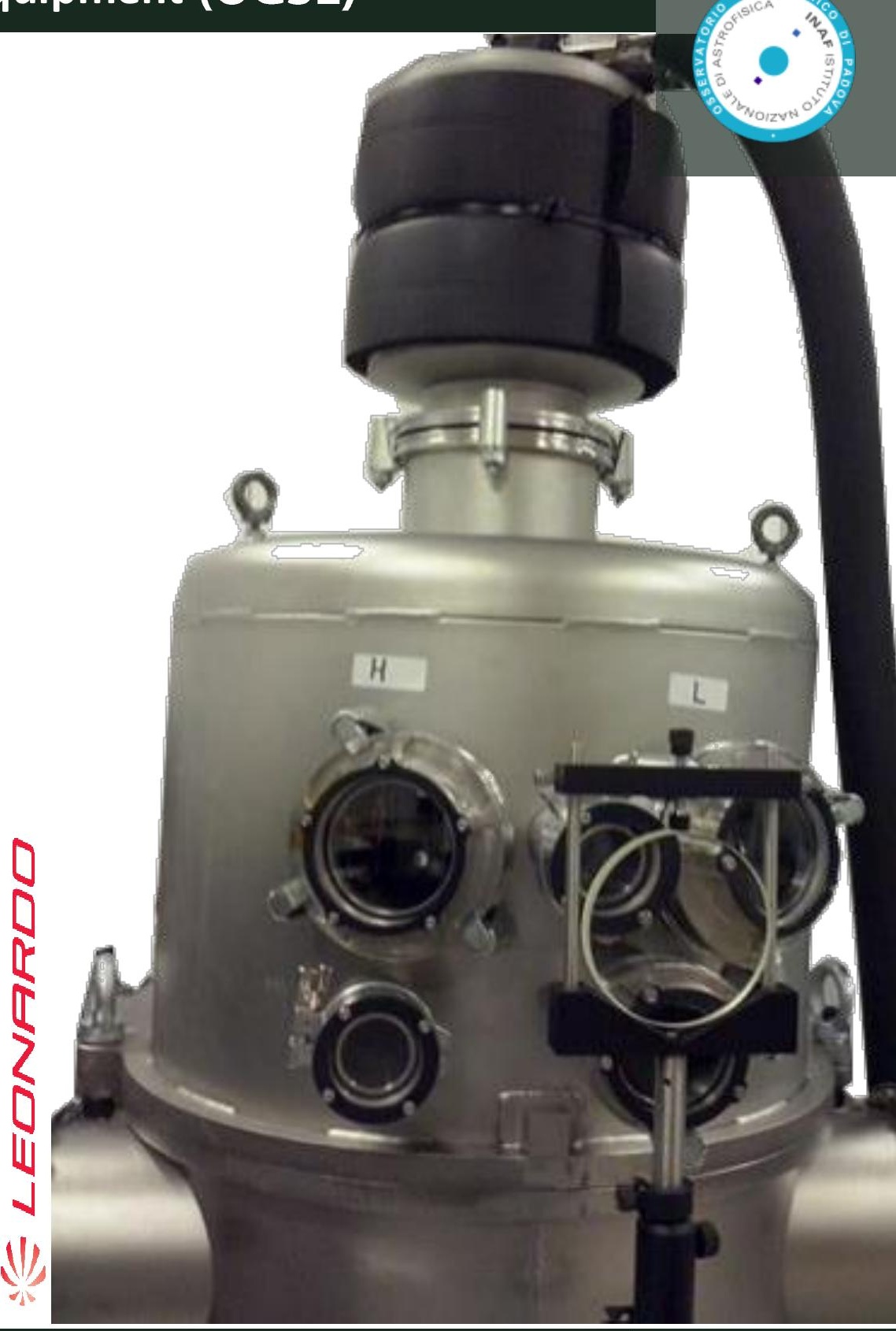




\section{Rational Model (18 DOF)}

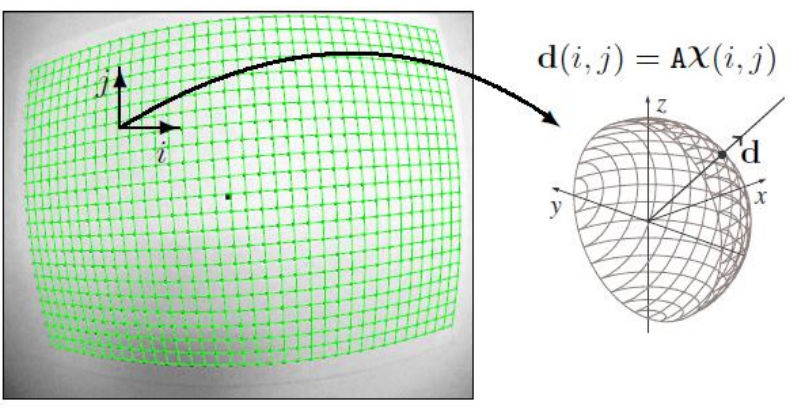

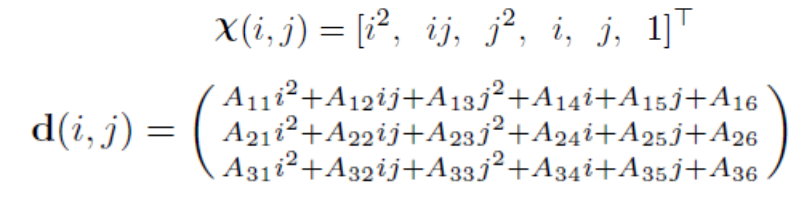

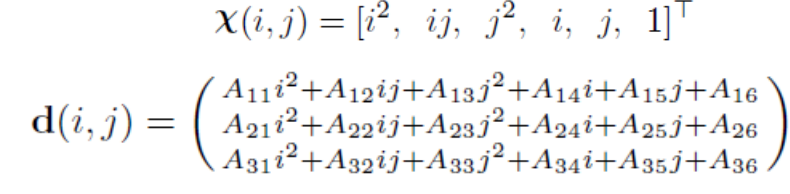

$$
\begin{aligned}
& \mathbf{d}(i, j)=\mathrm{A} \chi(i, j) \\
& d(i, j)=\mathrm{A} \chi(i, j)
\end{aligned}
$$

Distortion:

Temperature considered:

Distortion variation

Number of Spots:

Repetition:

Residuals:

10 Acq.

Distortion models will be soon updates in Spice Kernel definition.
In contrast to other existing models, which admit such linear estimates, the RFM is not specialized to any particular lens geometry, and is sufficiently general to model a variety of extreme distortions, as it will be demonstrated in this particular case. (Claus IEEE 2005)
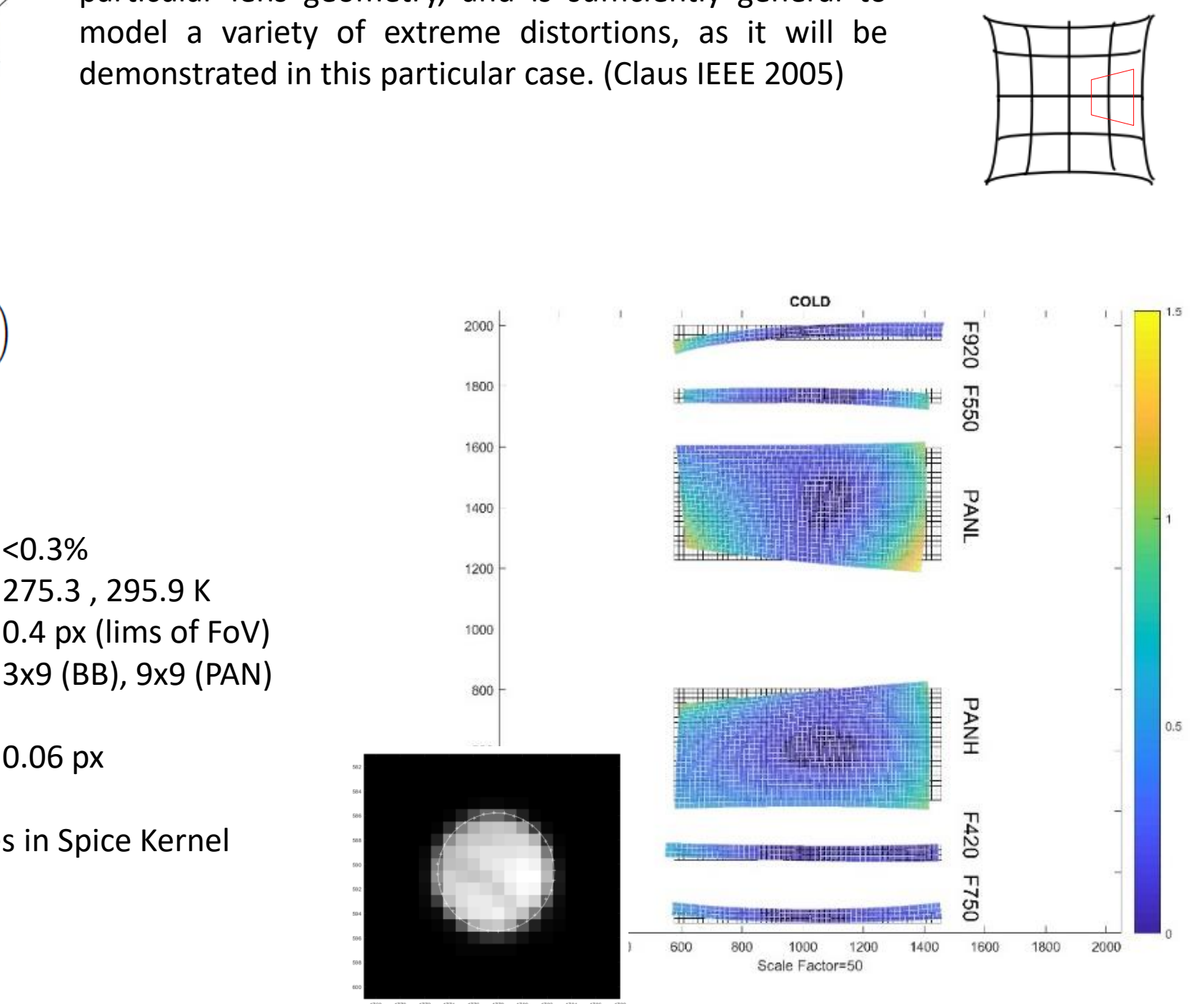

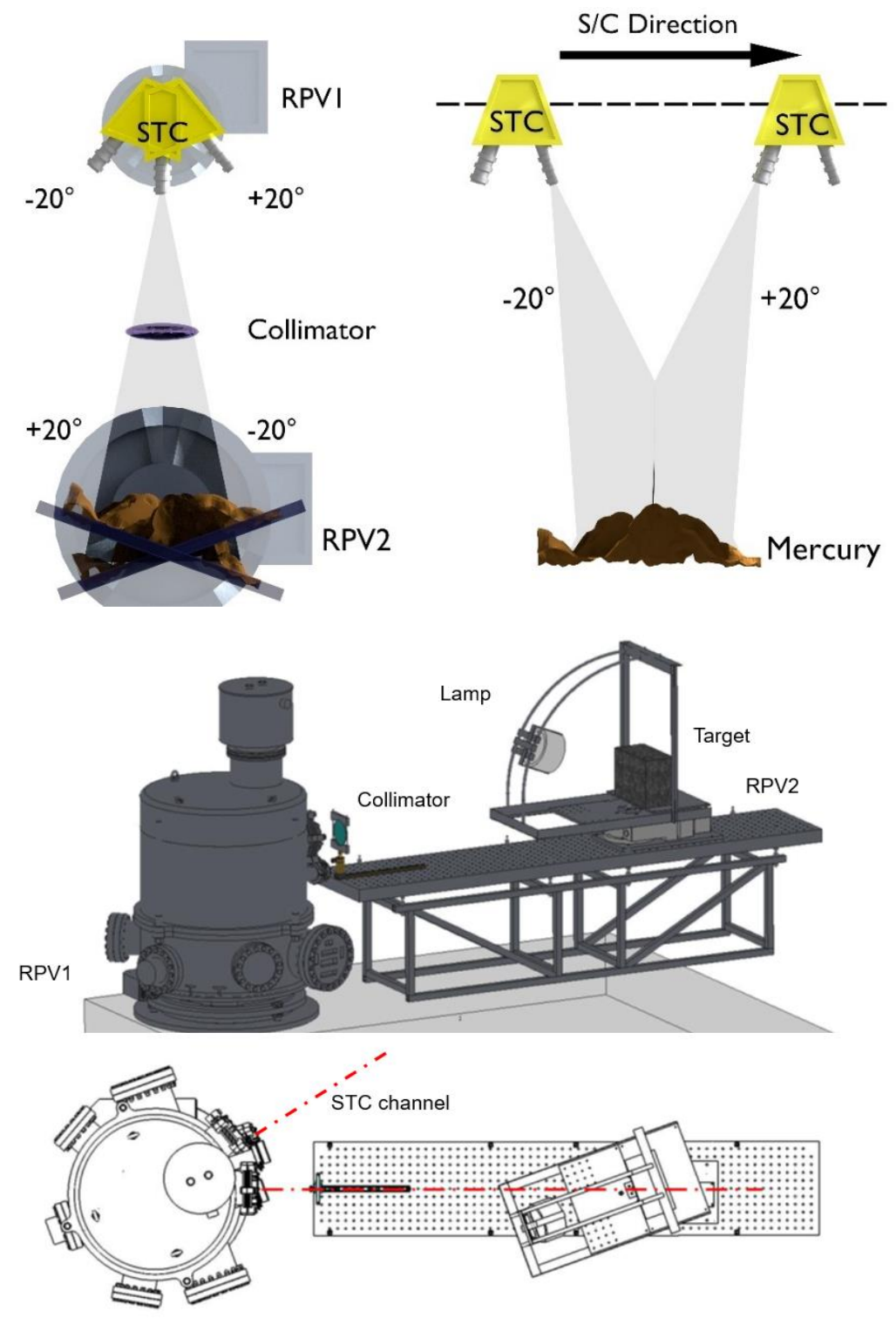

\section{SVS Concept}

During the calibration campaign of the telescope a Stereo Validation Setup has been conceived and realized in laboratory allowing to test the new push frame concept of STC.

- 2 RPV Rotational stage (Newport RV120-PE): bidirectional repeatability of about $0.002 \mathrm{deg}$.

- A collimator lens (JML DBL21597): achromatic doublet with nominal focal length of $1 \mathrm{~m}$ and a diameter of $80 \mathrm{~mm}$. Zemax simulations demonstrated distortion limited to 2.5 micrometres.

- Illumination source (solidal to RPV)

- Different Target (Anorthosite, Basalt, Concrete)

- $\mathrm{CAM}^{\circledR}$ FaroArm Platinum scanner reference DTMs

- GCP and Zhang for extrinsic parameter measurement.
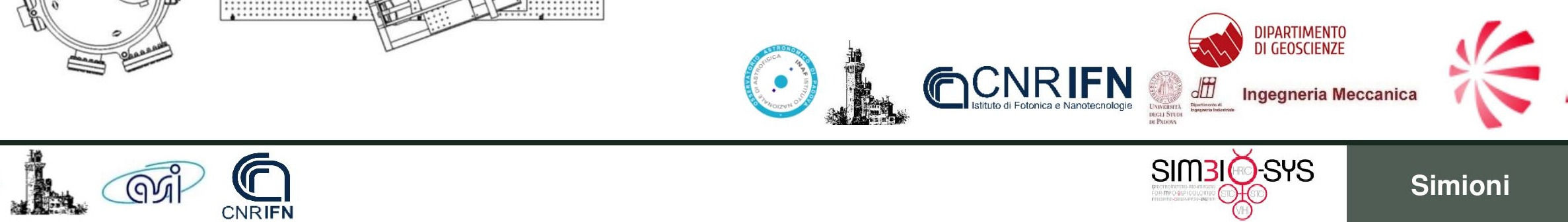


\section{Validation}

DTM are generated by the Stereo Reconstruction Pipeline. GCP alignment (corrected by ICP) allows the comparison with Laser scanner DTM [VP(Vertical Precision) $0.02 \mathrm{~mm}$ ].

Results show a VP of $73 \mu \mathrm{m}$ which corresponds to $36 \mathrm{~m}$ on STC first orbit (@480km).

\begin{tabular}{|l|c|}
\hline Data & STC-DTM \\
\hline Reference & Laser Acq \\
Threshold & $0.5 \mathrm{~mm}$ \\
\# Completeness & $99.6 \%$ \\
Mean & $1.9 \mu \mathrm{m}$ \\
RMS & $73 \mu \mathrm{m}$ \\
\hline
\end{tabular}
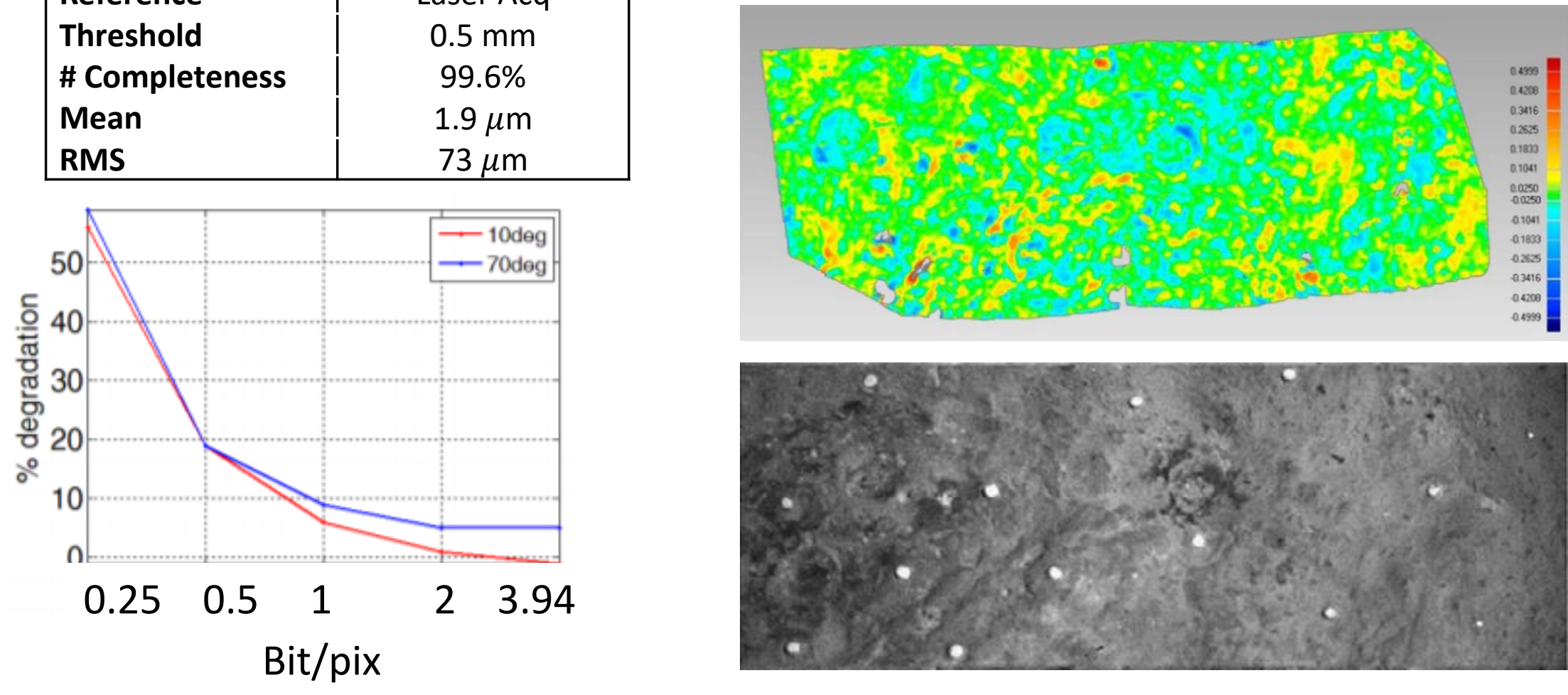


\section{Questions?}




\section{Backup slides}




\section{DTMs from MESSENGER datasets}

from laser altimeter (MLA) to MDIS derived stereo-topography
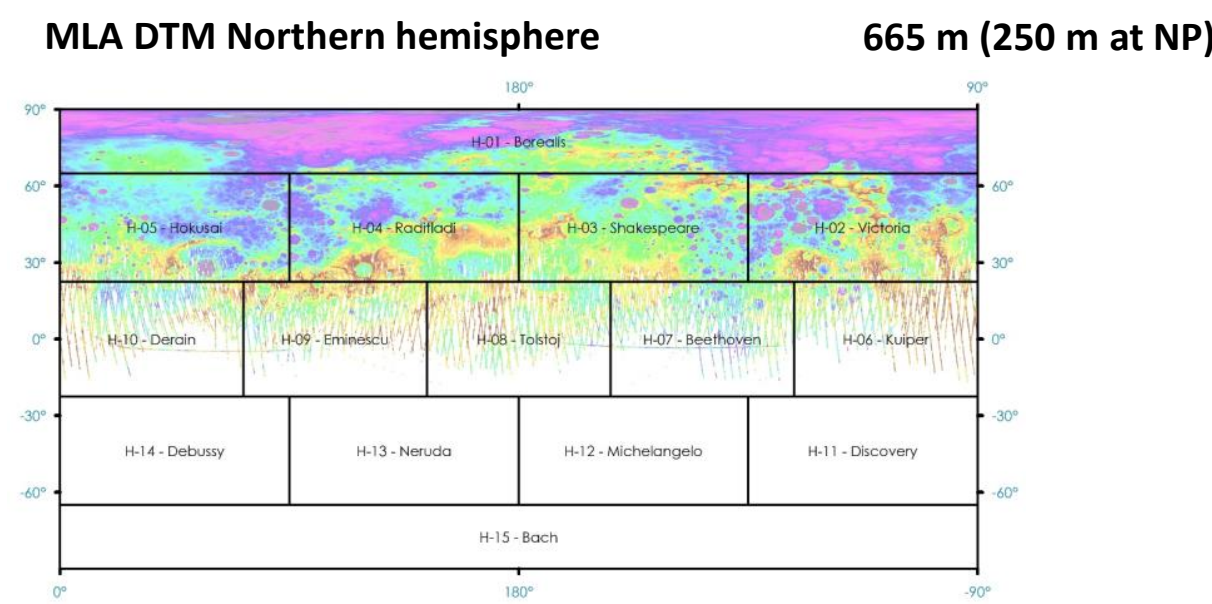

DLR regional stereo DTMs

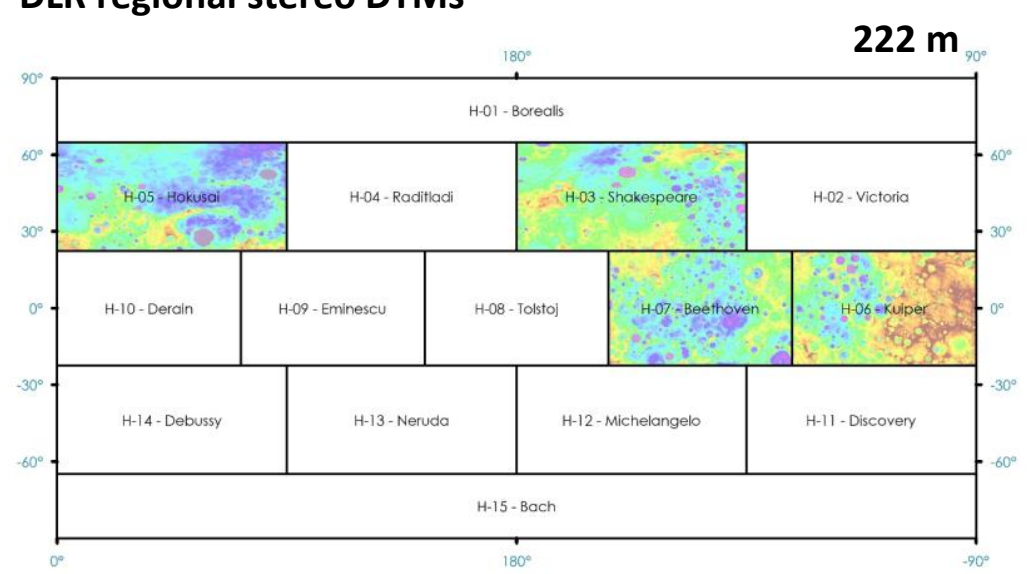

[Preusker et al., 2016]
USGS global multi-image DTM

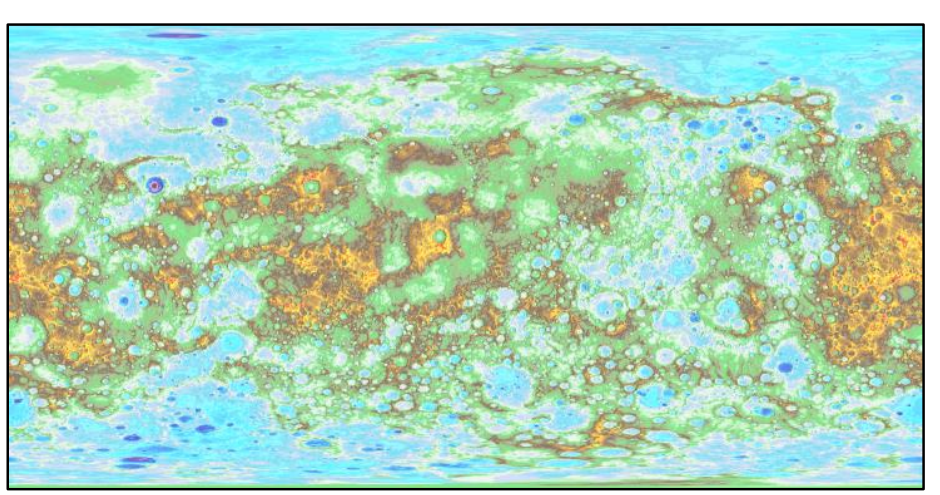

[Becker et al., 2016] 